\title{
Thymosin- $\beta 4$ Mediates Hepatic Stellate Cell Activation by Interfering with CircRNA-0067835/miR-155/FoxO3 Signaling Pathway
}

\author{
Lili Zhu ${ }^{\mathrm{a}, \mathrm{c}}$ Tingting Ren ${ }^{\mathrm{a}}$ Zixin Zhu ${ }^{\mathrm{b}} \quad$ Mingliang Chenga ${ }^{\mathrm{a}}$ Qiuju Mou \\ Mao Mua Yongmei Liu ${ }^{a} \quad Y^{a}$ umei Yao ${ }^{a} \quad$ Yiju Cheng ${ }^{a}$ Baofang Zhanga ${ }^{a}$ Zhuo Cheng ${ }^{d}$ \\ aDepartment of Infectious Diseases, The Affiliated Hospital of Guizhou Medical University, Guizhou, \\ ${ }^{b}$ Guizhou Medical University, The Affiliated Hospital of Guizhou Medical University, Guizhou, 'The \\ Affiliated Baiyun Hospital of Guizhou Medical University, Guizhou, dPeking University Health Science \\ Center, Beijing, China
}

\section{Key Words}

Liver fibrosis • Hepatic stellate cell • Thymosin $\beta 4$ • Circular RNA • MicroRNA • CeRNA

\begin{abstract}
Background/Aims: Hepatic stellate cells (HSCs) are the primary cell type responsible for liver fibrosis. Our study proved that thymosin beta 4 (Tß4) has anti-fibrogenic effects in HSCs through PI3K/AKT pathway. However, the underlying mechanisms are not fully elucidated. Circular RNAs (circRNAs) play important roles in fine-tuning gene expression and are often deregulated in cancers. However, the expression profile and clinical significance of in liver fibrosis is still unknown. Therefore, we hypothesize that T $\beta 4$ influences circRNAs in liver fibrosis. Methods: Circular RNA microarray was conducted to identify T $\beta 4$-related circRNAs. Pathway analysis and miRNA response elements analysis was conducted to predict the potential roles of differentially expressed circRNAs in liver fibrosis. CCK8 assays and flow cytometric assays were conducted to clarify the role of circRNA in liver fibrosis. Bioinformatics analysis and in vitro experiments were conducted to clarify the mechanism of circRNA-mediated gene regulation in liver fibrosis. Results: A total of 644 differentially expressed circRNAs were identified between the T $\beta 4$-depleted LX-2 cells and the control LX2 cells. The expression of circRNA-0067835 was significantly increased in the T $\beta 4$-depleted LX-2 cells compared with control. Knockdown of circRNA- 0067835 observably decreased LX-2 cell proliferation by causing G1 arrest and promoting apoptosis. Bioinformatics online programs predicted that circRNA-0067835 acted as miR-155 sponge to regulate FOXO3a expression, which was validated using luciferase reporter assay. Conclusion: Our experiments showed that circRNA-0067835 regulated liver fibrosis progression by acting as a sponge of miR-155 to promote FOXO3a expression,
\end{abstract}

L. Zhu and T. Ren contributed equally to this work and thus should be considered as co-first authors.

Mingliang Cheng

Department of Infectious Diseases, Affiliated Hospital of Guizhou

Medical University, 28 Guiyi Street, Guiyang, Guizhou 550004 (China)

E-Mail drerlisun@yeah.net 


\section{Cellular Physiology Cell Physiol Biochem 2018;51:1389-1398 \begin{tabular}{ll|l} 
and Biochemistry Published online: 27 November 2018 & $\begin{array}{l}\text { (c) } 2018 \text { The Author(s). Published by S. Karger AG, Basel } \\
\text { www.karger.com/cpb }\end{array}$ \\
\hline
\end{tabular} \\ Zhu et al.: CircRNA-0067835 and Liver Fibrosis}

indicating that circRNA-0067835 may serve as a potential therapeutic target for patients with liver fibrosis.

\section{Introduction}

Progression of liver fibrosis commonly occurs in virtually all types of chronic liver diseases (CLDs) [1]. During liver fibrosis, several complex processes occur, including excessive accumulation and hyperplasia of extracellular matrix proteins. Hepatic stellate cells (HSCs), the major source of fibrous matrix production, are the primary effector cell in the liver fibrosis [2]. Upon acute liver injury, quiescent HSCs become activated and transform into a myofibroblast-like phenotype characterized by secreting excess extracellular matrix (ECM) and expressing $\alpha$-SMA [3]. Therefore, the therapeutic antifibrogenic strategy for the modulation function of activated HSCs is very desirable.

Thymosin $\beta 4$ (T $\beta 4$ ), a highly conserved 43 - amino acid peptide, identified as the main intracellular G-actin sequestering protein that has a wide range of extracellular functions [4]. In recent papers, $\mathrm{T} \beta 4$ has been reported to act as anti-inflammatory and anti-fibrotic agents in vitro and in vivo [5]. Emerging evidence suggests that $\mathrm{T} \beta 4$ is anti-fibrotic in cultured human HSCs. However, the underlying mechanisms of T $\beta 4$ in liver fibrosis are not fully elucidated. Circular RNAs (circRNAs) are recently identified as novel class of endogenous noncoding RNAs (ncRNAs) that is highly represented in the eukaryotic transcriptome [6-8]. CircRNAs could regulate the gene expression at the transcriptional or post-transcriptional level by acting as microRNA (miRNA) sponges [9]. Recent study revealed the expression profile and potential function of differentially expressed circRNAs in irradiated HSCs, however, the biological functions of circRNAs in inhibitory effects of T $\beta 4$ on liver fibrosis remain unclear.

In this study, based on circRNA microarray analysis, we found T $\beta 4$ significantly regulated circRNA-0067835 in liver fibrosis. Our experiments showed that circRNA-0067835 regulated liver fibrosis progression by acting as a sponge of miR-155 to promote FOXO3a expression, indicating that circRNA-0067835 may serve as a potential therapeutic target for patients with liver fibrosis.

\section{Materials and Methods}

\section{Cell lines}

LX-2, a well-characterized human HSCs line, and HEK-293 cells were obtained from China Center for Type Culture Collection (CCTCC, Wuhan, China) and maintained in DMEM supplemented with $10 \%$ fetal bovine serum and $1 \%$ penicillin-streptomycin at $37{ }^{\circ} \mathrm{C}$ in $5 \% \mathrm{CO}_{2}$. Primary $\mathrm{HSC}$ were isolated from normal C57BL/6 mice.

\section{CCl4 liver injury model}

Eight-week-old male C57BL/6J mice $(n=6)$ received intraperitoneal injection of $7 \mu \mathrm{L} / \mathrm{g}$ of $10 \% \mathrm{CCl} 4$ (Sigma-Aldrich, St. Louis, MO, USA, cat\# 289116) in olive oil two times weekly for six weeks. Meanwhile, mice $(n=6)$ treated with olive oil treatment were considered as the control mice. The animals were provided by the Experimental Animal Center of Guizhou Medical University. The animal experimental protocol was approved by the University Animal Care and Use Committee of Guizhou Medical University. Mice were sacrificed under anesthesia at the end of six weeks and the livers were removed for further analysis. The liver tissues were used for Masson staining by fixation with $10 \%$ formalin. Quantitative analysis for the Masson-positive area was calculated from five fields for each liver slice.

\section{Cell transfection}

The small interfering RNAs (siRNAs), miR-155 mimics, miR-155 inhibitors and negative controls used for cell transfection were synthesized by GenePharma Co. Ltd. (Shanghai, China). The siRNAs and miRNAs were transfected into lung cancer cells using Lipofectamine 2000 Reagent (Life Technologies). 


\section{Cellular Physiology Cell Physiol Biochem 2018;51:1389-1398

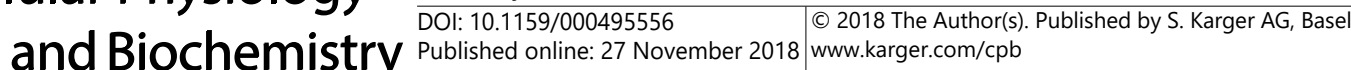 \\ Zhu et al.: CircRNA-0067835 and Liver Fibrosis}

\section{Expression profile analysis of circRNAs}

T $\beta 4$ siRNA transfected LX-2 cells were used for circRNA microarray. The circRNAs chip (ArrayStar Human circRNAs chip; ArrayStar, Rockville, MD, USA) containing 5, 639 probes specific for circular RNAs splicing sites was used. Following hybridization and washing, samples were analyzed on the circRNAs chips. Exogenous RNAs developed by the External RNA Controls Consortium (ERCC) were used as controls. circRNAs were enriched by digesting linear RNA with RNase R (Epicentre, Madison, WI, USA). Labeled RNAs were scanned using a Agilent Scanner G2505C (Agilent Technologies, Santa Clara, CA, USA). The circRNA microarray process was performed by KangChen Biotech, Inc. (Shanghai, China).

\section{Bioinformatics analysis}

The online target-predicting database miRBase (http://www.mirbase.org/) was used for the prediction of potential targeted sequences between circRNA-0067835 and miR-155. Another two databases, TargetScan (http://www.targetscan.org/) and miRanda (http://www.microrna.org/microrna/home.do), were used for the prediction of potential targeted sequences between miR-155 and the FOXO3a gene.

\section{Quantitative Real-Time PCR}

Total RNA and miRNAs were isolated using Trizol reagent (Invitrogen) and mirVana miRNA isolation kit (Ambion, Austin, TX, USA). Subseequently, cDNA was synthesized and amplified by RT-qPCR based on the TaqMan method on an ABI PRISM 7500 Sequence Detection System (Life Technologies, Grand Island, NY, USA) with the housekeeping gene GAPDH or U6 as an internal control. Relative expression levels of the genes were calculated using the $2^{-\Delta \Delta \mathrm{Ct}}$ method.

\section{Cell proliferation}

Cells were cultured to the logarithmic phase and then inoculated onto 96-well plates ( $10^{4}$ cells/well) for $24 \mathrm{~h}$. Cells were cultured for $24 \mathrm{~h}$ after treatment, cell proliferation was detected using the Cell Counting Kit-8 (CCK-8) reagent kit (Dojindo Molecular Technologies, Inc., Shanghai, China).

\section{Detection of cell cycle}

Cells were inoculated onto a 6 -well plate at $5 \times 10^{5}$ cells/well. After treatment, the cells were collected and washed twice with pre-cooled PBS. Cells were fixed in pre-cooled $75 \%$ ethanol in a $4^{\circ} \mathrm{C}$ refrigerator overnight, washed with PBS twice, and stained with propidium iodide (PI) containing RNase in the dark for 30 min. Cell cycle phase was detected using a flow cytometer (FACS420, BD Biosciences, San Jose, CA).

\section{Detecting apoptosis by flow cytometry}

An annexin V-allophycocyanin (APC)/ 4'6-diamidino-2-phenylindole (DAPI) double staining kit (Thermo Fisher Scientific) was used to analyze cellular apoptosis. Cells were seeded in 6-well plates $(5 \times 105$ cells/well) and treated with $1.0 \mathrm{mg} / \mathrm{L}$ adriamycin. Cells were then digested with trypsin (Gibcoß trypsinEDTA, Thermo Fisher Scientific), washed with PBS three times, suspended in $500 \mu \mathrm{l}$ of binding buffer, and then incubated with $5 \mu \mathrm{l}$ of FITC-conjugated annexin $V$ and $3 \mu$ of PI for $15 \mathrm{~min}$ at room temperature in the dark. The stained cells were detected using the BD FACS Aria II flow cytometer (BD Biosciences, Hercules, CA, USA).

\section{Assay of lactate dehydrogenase (LDH) release}

LX-2 cells were seeded in 96-well plates at a density of $5 \times 10^{3}$ cells/well. The cells were incubated in DMEM supplemented with $10 \%$ fetal bovine serum and $1 \%$ penicillin-streptomycin at $37{ }^{\circ} \mathrm{C}$ in $5 \% \mathrm{CO}_{2}$ for $24 \mathrm{~h}$. Then cells were synchronized with serum starvation for another $24 \mathrm{~h}$. After starvation, LX-2 cells were treated with DMSO or ATG at certain concentrations for 48h. LDH activities in culture medium were investigated by measuring the A value at $440 \mathrm{~nm}$ using a LDH release assay kit (Nanjing Jiancheng Bioengineering Institute, Nanjing, China) according to the manufacturer's instructions. 


\section{Cellular Physiology Cell Physiol Biochem 2018;51:1389-1398 \begin{tabular}{ll|l} 
and Biochemistry & Published online: 27 November 2018 & $\begin{array}{l}\text { (c) } 2018 \text { The Author(s). Published by S. Karger AG, Basel } \\
\text { www.karger.com/cpb }\end{array}$ \\
\hline
\end{tabular} \\ Zhu et al.: CircRNA-0067835 and Liver Fibrosis}

\section{Luciferase activity assays}

Cells were co-transfected with circRNA-0067835 plasmids or their mutant fragments and miR-155 mimic by using Lipofectamine 2000 (Invitrogen, Foster city, CA) according to the manufacturer's protocol. Firefly and Renilla luciferase activities were measured consecutively using a Dual-Luciferase Reporter Assay System (Promega, Massachusetts, USA) after transfection for $48 \mathrm{~h}$. Each assay was repeated in six independent experiments.

\section{Western Blot Analysis}

Protein extracts were run on SDS acrylamide gels and transferred onto nitrocellulose. Blots were incubated with anti-Akt, anti-FoxO3a, and GAPDH (Cell Signaling Technology, Beverly, MA, USA) overnight at $4^{\circ} \mathrm{C}$. After incubation with secondary horseradish-peroxidase conjugated antibodies (Santa Cruz Biotechnology), the bands were visualized by the enhanced chemiluminescence light method (Amersham Biosciences) and exposed to X-omat film (Eastman Kodak Co., New Haven, CT) or a chemiluminescence imager (Image Station 2000R, Eastman Kodak Co.).

\section{Statistical Analysis}

All data are reported as the mean \pm S.D. Statistical significance was determined by Student's t-tests using the SPSS19 software program when comparisons were made between two different groups.

\section{Results}

Analysis of circRNA expression profiles in LX-2 cells after inhibition of T $\beta 4$

To investigate whether T $\beta 4$ regulates circRNA expression in LX-2 cells, we knockdown the levels of T $\beta 4$ in LX-2 cells by T $\beta 4$-specific siRNA (T $\beta 4$ siRNA). To identify specific circRNAs that are differentially expressed between T $\beta 4$ siRNA and control samples, three pairs of T $\beta 4$ siRNA samples and control samples were subjected for circRNA microarray assay. A total of 644 circRNAs that were differently expressed by $>3$ fold between the T $\beta 4$ siRNA groups and the control were identified. Subsequently, we narrowed the scope of the analysis to the 20 most aberrantly expressed circRNAs, including 10 upregulated circRNAs and 10 downregulated circRNAs (shown in the heatmap in Fig. 1A). We selected 10 mostly dysregulated circRNAs for validation using real-time PCR in patients with liver fibrosis, general consistency was shown between the high-throughput data and real-time PCR results. Among these circRNAs, the expression of circRNA-0067835 was significantly increased in the T $\beta 4$ depleted LX-2 cells compared with the control cells $(P<0.01$, Fig. 1B). Moreover, we observed significantly increased expression of circRNA-0067835 in HSCs from the livers of healthy mice cultured for 7 days than freshly isolated cells $(P<0.01$, Fig. 1C). Besides, circRNA-0067835 expression was analyzed in CCl4-induced liver fibrosis in mice, and the results showed that the expression of circRNA-0067835 in CCl4-treated liver was markedly increased as compared with control liver $(P<0.01$, Fig. 1D), indicating that there are associations between circRNA-0067835 and liver fibrosis.

\section{Effects of circRNA-0067835 on LX-2 cells}

The qRT-PCR results presented that circRNA-0067835 expression in LX-2 cells was remarkably decreased after transfection with si-circRNA-0067835 (Fig. 2A, $P<0.01$ ). Furthermore, CCK-8 assay confirmed that knockdown of circRNA-0067835 observably suppressed LX-2 cell proliferation (Fig. $2 \mathrm{~B}, P<0.01$ ). In addition, flow cytometry analysis disclosed the variance of cell cycle and apoptosis condition after transfection with sicircRNA-0067835. As exhibited in Fig. 2C, the percentage of cells arrested in G0/G1 phase was significantly higher in si-circRNA-0067835 group than in NC group, while that of arrested cells in $\mathrm{S}$ phase relatively declined compared with $\mathrm{NC}$ group $(P<0.01)$. Meanwhile, the apoptosis rate of LX2 cells significantly increased after transfection of si-circRNA-0067835 


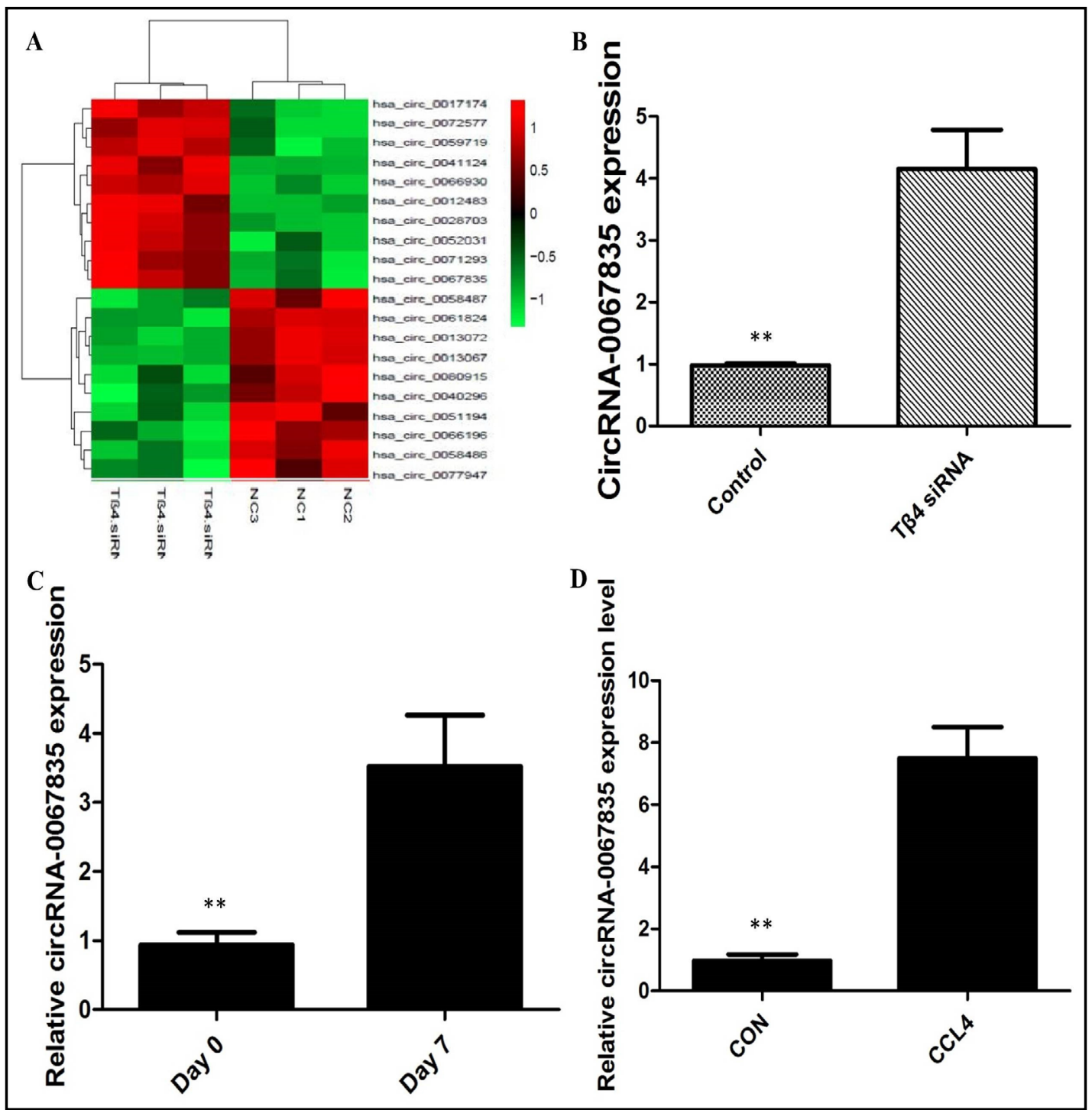

Fig. 1. (A) Differential expression of selected circRNAs in T $\beta 4$-depleted LX-2 cells and the control LX2 cells. Based on the circRNA microarray results, the top 10 upregulated and top 10 downregulated circRNAs in T 34 -depleted LX-2 cells and the control LX2 cells are shown in the heatmap. The red colour represents high expression, whereas the green colour represents low expression. (B) qRT-PCR verification of the expression of circRNA-0067835 in T 34 -depleted LX-2 cells and the control LX2 cells. (C) qRT-PCR verification of the expression of circRNA-0067835 in HSCs from the livers of healthy mice cultured for 7 days than freshly isolated cells. (D) The qRT-PCR results presented that circRNA-0067835 expression was remarkably increased in CCl4-treated liver as compared with control liver.

(Fig. 2D-E, $P<0.01$ ). Overall, inhibition of circRNA-0067835 arrested cell propagation and cell cycle and promoted apoptosis in LX2 cells. We evaluated the cell toxicity by LDH release and found that there is no significant LDH leakage in the LX-2 cells after transfection of sicircRNA-0067835 (Fig. 2F).

\section{CircRNA-0067835 functions as a miRNA sponge for miR-155 in liver fibrosis}

As circRNAs function mainly as miRNA sponges to bind to functional miRNAs and then regulate gene expression, we then examined the potential miRNAs associated with circRNA-0067835. According to bioinformatics analysis using miRBase (http://www. mirbase.org/), circRNA-0067835 possessed a complementary sequence to the miR-155 


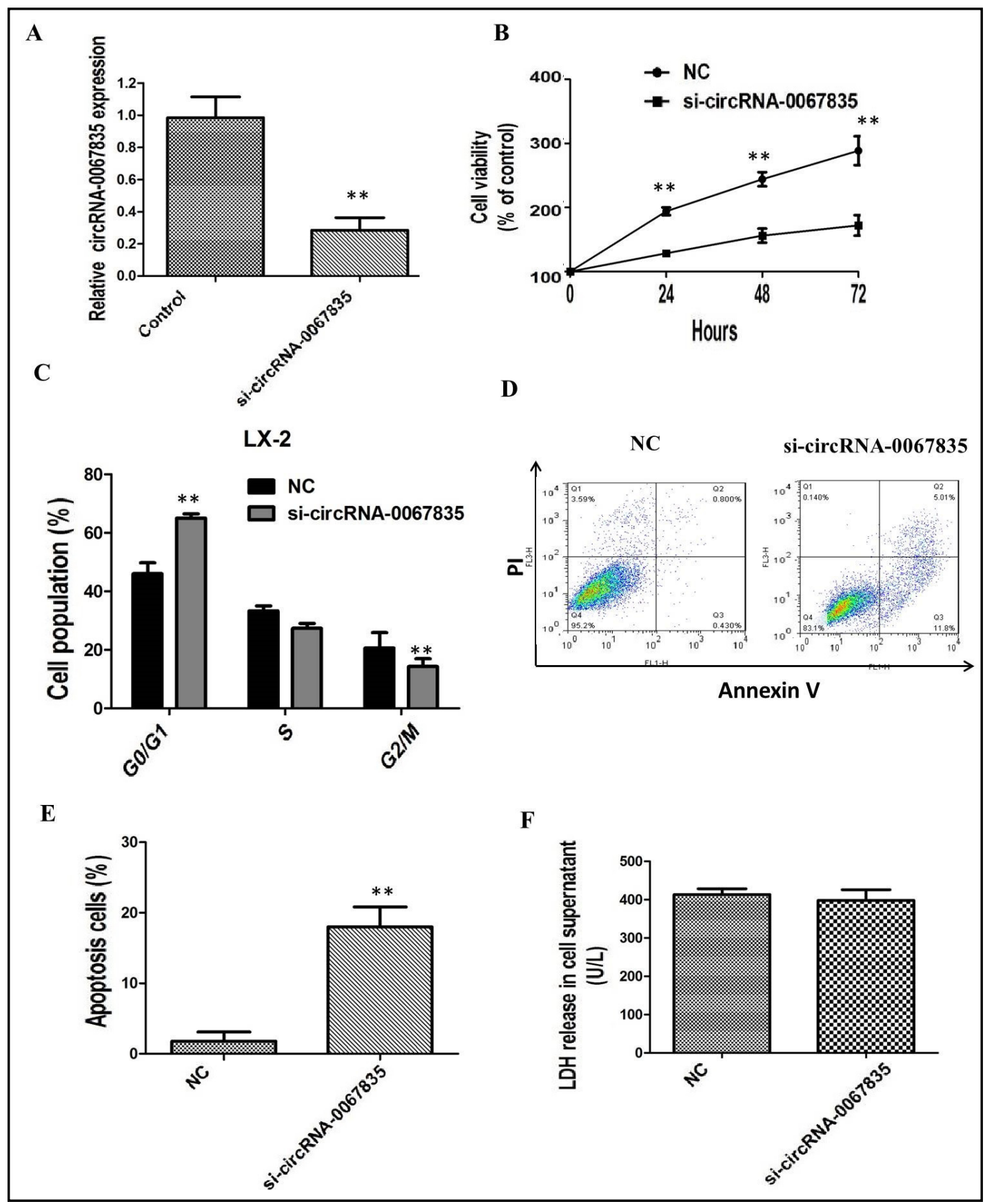

Fig. 2. (A) The qRT-PCR results presented that circRNA-0067835 expression in LX-2 cells was remarkably decreased after transfection with si-circRNA-0067835. (B) CCK8 assay showing knockdown of circRNA-0067835 observably suppressed LX-2 cell proliferation. (C) The flow cytometry assay showed that knockdown of circRNA-0067835 had cell-cycle arrest at the G1-G0 phase in LX-2 cells. (D-E) The flow cytometry assay showed that knockdown of circRNA-0067835 had higher apoptotic rate in LX-2 cells. (F) There is no significant LDH leakage in the LX-2 cells after transfection of si-circRNA-0067835.

seed region. To confirm whether circRNA-0067835 directly binds to miR-155, we performed dual-luciferase reporter assay. The results displayed that luciferase activity was repressed in LX2 cells co-transfected with wild-type (WT) circRNA-0067835 and miR-155 mimics while restored in cells cotransfected with mutant (Mut) circRNA-0067835 and miR-155 mimics (Fig. 3A). Moreover, knockdown of circRNA-0067835 significantly promoted miR-155 
Fig. 3. (A) The luciferase activity was repressed in LX-2 cells co-transfected with wild-type (WT) c i r c R N A - 0067835 and miR-155 mimics while restored in cells cotransfected with mutant (Mut) cir c R N A - 0067835 and miR-155 mimics. (B) Knockdown of c i r c R N A - 0067835 significantly increased miR-155 expression in LX2 cells. (C) The qRTPCR results presented that miR-155 expression was remarkably decreased in HSCs from the livers of healthy mice cultured for 7 days than freshly isolated cells. (D) The qRT-PCR results presented that miR-155 expression was remarkably decreased in CCl4-treated liver as compared with control liver.

Fig. $\quad$ 4. (A) Bioinformatics analysis revealed the predicted binding sites between FOXO3 and miR-155. (B) Luciferase reporter assay demonstrated miR-155 mimics significantly decreased the luciferase activity of F0X03-wt in HEK293T cells. (C) Overexpression of miR-155 inhibited the expression of FOXO3a in LX-2 cells. (D) Inhibition of miR-155 increased the expression of FOX03a in LX-2 cells. (E) Overexpression of miR-155 inhibited the expression of AKT in LX-2 cells. (F) Inhibition of miR155 increased the expression of AKT in LX-2 cells.

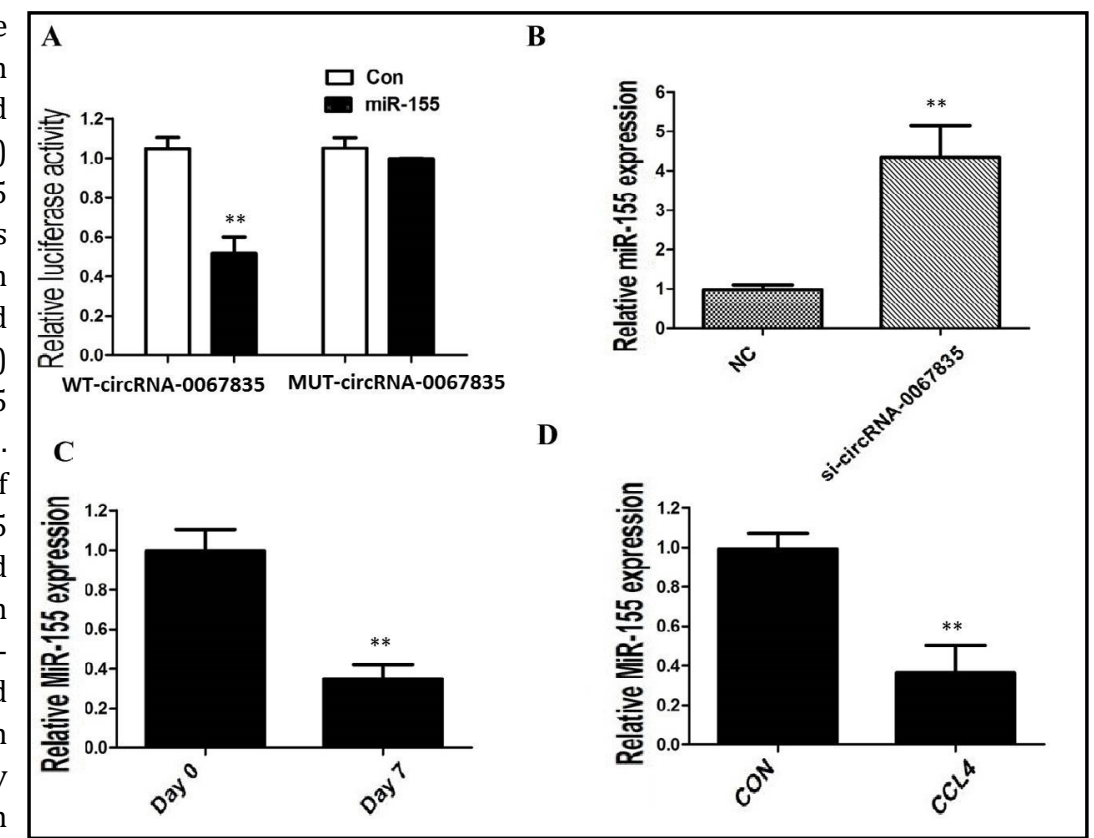

B 


\section{Cellular Physiology Cell Physiol Biochem 2018;51:1389-1398

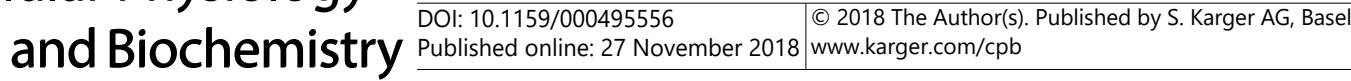

Fig. 5. (A) The expression of FOXO3a and AKT in HSCs from the livers of healthy mice cultured for 7 days than freshly isolated cells. (B) The expression of FOXO3a and AKT in CCl4-treated liver as compared with control liver. (C) Knockdown of T $\beta 4$ expression upregulated the expression of FOXO3a and AKT in LX-2 cells. (D) T $\beta 4$ treatment significantly inhibited the expression of FOXO3a and AKT.

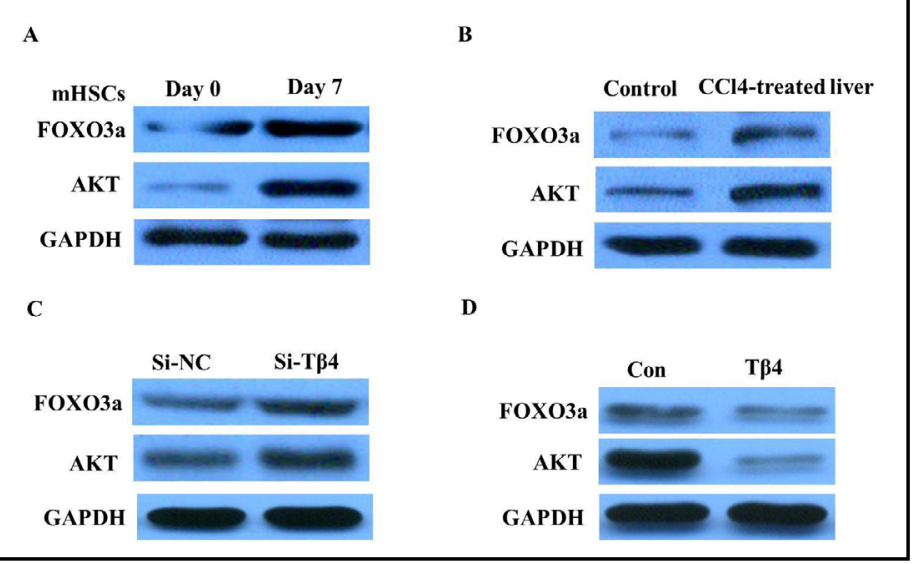

A

expression in LX2 cells (Fig. 3B). MiR-155 expression was also studied in HSCs and CCl4treated liver, and the results of RT-qPCR also revealed that a statistically decrease compared to controls $(P<0.01$, Fig. 3C-D).

miR-155 exerts its role by regulation of FOXO3 a expression in liver fibrosis We further explored the mechanism by which miR-155 participated in liver fibrosis, potential target genes for miR-155 were therefore searched for using bioinformatics software. On the basis of miRNA target analysis, FOXO3a is a potential target mRNA of miR-155 (Fig. 4A). To prove it, we performed luciferase reporter assay to validate the interaction between miR-155 and the 3'UTR of FOXO3a mRNA. The results indicated that luciferase activity was reduced in HEK293 cells co-transfected with FOXO3a-WT and miR-155 mimics and restored in cells co-transfected with FOXO3a-Mut and miR-155 mimics (Fig. 4B). As shown in Fig. 4C-D, the expression level of FOXO3a was significantly decreased in miR-155-overexpressing cells and increased in miR-155-silenced cells. AKT kinase is known to play a key role in phosphorylating and repressing FOXO3a transcriptional activity. As predicted, overexpressing miR-155 drastically decreased the levels of AKT, and silencing miR-155 increased AKT expression (Fig. $4 \mathrm{E}-\mathrm{F}$ ), suggesting that miR-155 contributed to the modulation of AKT/FOXO3a signaling. The expression of FOXO3a and AKT were significantly increased in HSCs and CCl4-treated liver, indicating that there are associations between AKT/FOXO3a signaling and liver fibrosis (Fig. 5A-B).

\section{Thymosin- $\beta 4$ mediates hepatic stellate cell activation through AKT/FOXO3a signaling}

To investigate whether T $\beta 4$ regulates AKT/FOXO3a pathway in LX-2 cells, we knockdown the levels of T $\beta 4$ in LX- 2 cells by T $\beta 4$ siRNA. As expected, the expression of FOXO3a and AKT can be increased in the T $\beta 4$-depleted LX-2 cells $48 \mathrm{~h}$ post-transfection compared with the Con siRNA (Fig. 5C). Then, we treated LX-2 cells with T $\beta 4(100 \mathrm{ng} / \mathrm{mL})$ and then incubated for 24 hours. Then, we examined the level of AKT/FOXO3a pathway. Western blot analysis revealed significantly reduced expression of Akt and FOXO3a expression at the protein level by the treatment of T $\beta 4$ (Fig. 5D).

\section{Discussion}

Liver fibrosis, characterized by abnormalities in the hepatic structure, is the excessive accumulation and hyperplasia of extracellular matrix proteins. As shown in recent research, $\mathrm{T} \beta 4$ served an important role in the occurrence and development of liver fibrosis. Depletion of T $\beta 4$ significantly promoted the migration, proliferation, and activation of HSCs by regulating the PI3K/AKT pathway. PI3K signaling, one of the targets of T $\beta 4$, provides intense growth and survival signals $[10,11]$. The PI3K enzyme, as a well-known upstream mediator 


\section{Cellular Physiology Cell Physiol Biochem 2018;51:1389-1398

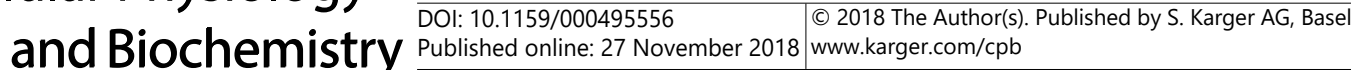 \\ Zhu et al.: CircRNA-0067835 and Liver Fibrosis}

of Akt kinase activation, could recruit Akt kinase to the membrane and activate Akt by phosphorylation. Activated Akt further phosphorylates several downstream proteins that play central roles in liver fibrosis [12].

As a novel gene regulator, circRNAs are potentially involved in multiple biological and pathological processes [13]. However, very little is known regarding their roles in liver fibrosis. In this study, we investigated the role of T $\beta 4$-related circRNAs in liver fibrosis. We found that 644 circRNAs that were differently expressed by $>3$ fold between the T $\beta 4$-depleted LX-2 cells and the control LX2 cells. Specifically, we observed significantly increased expression of circRNA-0067835 in HSCs from the livers of healthy mice cultured for 7 days than freshly isolated cells. Besides, circRNA-0067835 expression was markedly increased in CCl4-treated liver as compared with control liver. Moreover, knockdown of circRNA-0067835 arrested cell propagation and cell cycle and promoted apoptosis in LX-2 cells. Specifically, we also showed mechanistically that circRNA-0067835 regulates the progression of liver fibrosis by inhibiting miR-155 expression. In conclusion, evidence for circRNA-0067835 functionality in liver fibrosis also extended to regulate AKT/FOXO3a signaling.

Since the first report of circRNA functioning as a miRNA sponge, the potential of circRNAs in regulating cancer-related genes through fine-tuning miRNAs has recently been recognized $[14,15]$. In this study, miR-155 showed a complementary sequence to circRNA-0067835 based on bioinformatics analysis, and this miRNA was finally identified as the endogenous competing RNA by luciferase reporter assay. Moreover, enhanced expression of circRNA-0067835 significantly suppressed miR-155 expression in LX-2 cells. We showed that the expression of miR-155 was downregulated in HSCs and CCl4-treated liver, indicating that circRNA-0067835 may play an important role through the sponge activity of miR-155.

The FoxO family of Forkhead transcription factors has been reported to regulate HSCs proliferation via the PI3K/Akt pathway $[16,17]$. AKT kinase is known to play a key role in phosphorylating and repressing FOXO3a transcriptional activity. FOXO3a plays a key role in integrating multiple pathways. FOXO3a proteins play an important role in maintaining intracellular redox balance and biological survival under different environmental stresses [18]. In the present study, FOXO3a gene was then identified as a direct target of miR-155 by performing bioinformatics analysis and subsequent luciferase reporter assay. The expression level of FOXO3a and AKT were significantly decreased in miR-155-overexpressing cells and increased in miR-155-silenced cells, suggesting that miR-155 contributed to the modulation of AKT/FOXO3a signaling. The expression of FOXO3a and AKT were significantly increased in HSCs and CCl4-treated liver, indicating that there are associations between AKT/FOXO3a signaling and liver fibrosis. Western blot analysis revealed knockdown T $\beta 4$ increased the expression of FOXO3a and AKT, and treatment of T $\beta 4$ significantly reduced expression of FOXO3a and AKT, suggesting that T $\beta 4$ was involved in HSCs activity through regulation of AKT/FOXO3a signaling.

\section{Conclusion}

Our current study demonstrates that circRNA-0067835 is an important modifier of pathogenesis of liver fibrosis by serving as a miRNA sponge for miR-155 and thereby promoting the function of FOXO3a signaling pathway. Our results can help to illuminate the molecular mechanisms underlying liver fibrosis and may potentially serve a novel therapeutic target for the treatment of liver fibrosis.

\section{Acknowledgements}

This work was financially supported by the National Nature Science Foundation of China (Grant No. 81570543). 


\section{Cellular Physiology Cell Physiol Biochem 2018:51:1389-1398

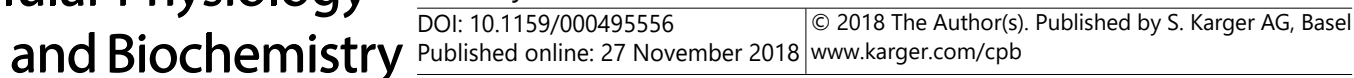 Zhu et al.: CircRNA-0067835 and Liver Fibrosis}

\section{Disclosure Statement}

No potential conflicts of interest were disclosed.

\section{References}

Friedman SL: Mechanisms of hepatic fibrogenesis. Gastroenterology 2008;134:1655-1669. Wang J, Chu ES, Chen HY, Man K, Go MY, Huang XR, Lan HY, Sung JJ, Yu J: microRNA-29b prevents liver fibrosis by attenuating hepatic stellate cell activation and inducing apoptosis through targeting PI3K/AKT pathway. Oncotarget 2015;6:7325-7338.

-3 Popov Y, Schuppan D: Targeting liver fibrosis: strategies for development and validation of antifibrotic therapies. Hepatology 2009;50:1294-1306.

4 Goldstein AL, Hannappel E, Kleinman HK: Thymosin beta4: actin-sequestering protein moonlights to repair injured tissues. Trends Mol Med 2005;11:421-429.

5 Huang HC, Hu CH, Tang MC, Wang WS, Chen PM, Su Y: Thymosin beta4 triggers an epithelial-mesenchymal transition in colorectal carcinoma by upregulating integrin-linked kinase. Oncogene 2007;26:2781-2790.

6 Chen BJ, Byrne FL, Takenaka K, Modesitt SC, Olzomer EM, Mills JD, Farrell R, Hoehn KL, Janitz M: Analysis of the circular RNA transcriptome in endometrial cancer. Oncotarget 2018;9:5786-5796.

7 Hansen TB, Jensen TI, Clausen BH, Bramsen JB, Finsen B, Damgaard CK, Kjems J: Natural RNA circles function as efficient microRNA sponges. Nature 2013;495:384-388.

-8 Salzman J, Chen RE, Olsen MN, Wang PL, Brown PO: Cell-type specific features of circular RNA expression. PLoS Genet 2013;9:e1003777.

-9 Zhou J, Xiong Q Chen H, Yang C, Fan Y: Identification of the Spinal Expression Profile of Non-coding RNAs Involved in Neuropathic Pain Following Spared Nerve Injury by Sequence Analysis. Front Mol Neurosci 2017;10:91.

10 Zheng J, Wu C, Xu Z, Xia P, Dong P, Chen B, Yu F: Hepatic stellate cell is activated by microRNA-181b via PTEN/Akt pathway. Mol Cell Biochem 2015;398:1-9.

11 Adachi M, Osawa Y, Uchinami H, Kitamura T, Accili D, Brenner DA: The forkhead transcription factor Fox01 regulates proliferation and transdifferentiation of hepatic stellate cells. Gastroenterology 2007;132:14341446.

12 Pan X, Zhang Y, Kim HG, Liangpunsakul S, Dong XC: FOXO transcription factors protect against the dietinduced fatty liver disease. Sci Rep 2017;7:44597.

13 Li J, Yang J, Zhou P, Le Y, Zhou C, Wang S, Xu D, Lin HK, Gong Z: Circular RNAs in cancer: novel insights into origins, properties, functions and implications. Am J Cancer Res 2015;5:472-480.

14 Han D, Li J, Wang H, Su X, Hou J, Gu Y, Qian C, Lin Y, Liu X, Huang M, Li N, Zhou W, Yu Y, Cao X: Circular RNA circMT01 acts as the sponge of microRNA-9 to suppress hepatocellular carcinoma progression. Hepatology 2017;66:1151-1164.

15 Zhong Z, Huang M, Lv M, He Y, Duan C, Zhang L, Chen J: Circular RNA MYLK as a competing endogenous RNA promotes bladder cancer progression through modulating VEGFA/VEGFR2 signaling pathway. Cancer Lett 2017;403:305-317.

16 Xu B, Wang G, Zhang J, Cao W, Chen X: Resveratrol decreases FoXO protein expression through PI3K-Aktdependent pathway inhibition in H(2)O(2)-treated synoviocytes. Histol Histopathol 2017:11884.

17 Kao YH, Chen PH, Wu TY, Lin YC, Tsai MS, Lee PH, Tai TS, Chang HR, Sun CK: Lipopolysaccharides induce Smad2 phosphorylation through PI3K/Akt and MAPK cascades in HSC-T6 hepatic stellate cells. Life Sci 2017;184:37-46.

18 Tu W, Ye J, Wang ZJ: Embryonic liver fordin is involved in glucose glycolysis of hepatic stellate cell by regulating PI3K/Akt signaling. World J Gastroenterol 2016;22:8519-8527. 